\title{
Multimodal molecular imaging: Insight into the complexity of biological surfaces through speed, resolution and identification.
}

\author{
Ron M.A. Heeren ${ }^{1,2}$, Anne L. Bruinen ${ }^{1}$, Nadine E. Mascini ${ }^{2}$, Gregory L. Fisher ${ }^{3}$, Tiffany Porta ${ }^{1,2}$ and \\ Shane R. Ellis ${ }^{1}$ \\ ${ }^{1}$ M4I, The Maastricht MultiModal Molecular Imaging Institute, University of Maastricht, Maastricht, \\ The Netherlands. \\ ${ }^{2}$ FOM-AMOLF, Science Park 104, 1098 XG Amsterdam, The Netherlands \\ ${ }^{3}$ Physical Electronics, 18725 Lake Drive East, Chanhassen MN, USA
}

The chemical complexity of biological surfaces is highly dynamic and subject to local changes in response to a changing environment. This chemical heterogeneity is a particular important parameter when considering treatment of diseases such as cancer. It is this inconceivably complex heterogeneity that makes tumors so difficult to treat as no single therapy targets all permutations of phenotypes and environment precisely. This implies that to make truly personalized tumor therapy reality a diagnostic method is needed that unravels this spatial and molecular complexity of tumor tissue.

Here, we will argue that multimodal imaging mass spectrometry [1] is a diagnostic and prognostic tool that can elucidate tissue (and tumor) molecular heterogeneity and employ it to predict or design therapy response. A molecular view at different spatial and molecular levels is required to realize this ambitious goal. A combination of immunohistochemistry, Secondary Ion Mass Spectrometry (SIMS) and Matrix Assisted Laser Desorption Ionization (MALDI) imaging mass spectrometry is employed to classify and stratify different cellular phenotypes as well as to predict therapy response. The distribution of several hundreds of molecules on the surface of complex (biological) surfaces can be determined directly in a single imaging MS experiment. This enables molecular pathway analysis as well as the investigation of the role of the different molecular signals and their behavior under influence of a drastically changing physical, chemical or biological environment.

Imaging MS is a label-free imaging technique that is capable of visualizing the molecular distribution on the surface of a biological sample. State-of-the-art molecular imaging mass spectrometry has evolved to bridge the gap between different disciplines such as MRI, PET, fluorescence imaging and histology. Among imaging MS modalities, SIMS is considered a high spatial resolution molecular imaging technique that addresses 'clean' predominantly unprepared tissue surfaces. MALDI on the other hand is considered to provide a broader molecular scope on biological thin tissue sections that have been covered with a thin layer of minute matrix crystals that assist desorption and ionization. A particular advantage of MALDI is the detection in a higher mass range and the ability to characterize biological compounds with routine tandem MS. SIMS has, for a long time, be devoid of tandem MS based identification capabilities. Here, we present a novel high resolution tandem TOF-SIMS method to analyze and identify intact lipid ions from thin tissue sections. This new approach enables simultaneous surface screening with MS1 and targeted identification with MS2.

The clinical acceptance of imaging MS greatly depends on the validation of the molecular images employed for patient and tissue classification. Modern pathology/hospital biobanks contain both chemically fixed (through formalin fixation and paraffin embedding) and cryopreserved material for 
large patient cohorts. Fundamental studies have, for a considerable amount of time, provided insight into signaling pathways of lipids, peptides and proteins directly from tissues of these biobanks. Their validation however was severely hampered by the lack of molecular imaging throughput. A number of innovative developments have emerged that significantly have increased the speed of analysis. We will demonstrate these advances based on a the rapid analysis of a number of different tumor models including breast cancer (BC) and cholangiocarcinoma (CCA).

The (macro)molecular information obtained with high throughput imaging MS is combined with routine histology (H\&E staining), allowing linking of molecular data with specific cellular structures. Molecular profiles of CCA will be correlated with (i) tumor (sub)class, i.e. intrahepatic vs. hilar/distal CCA, mucin-negative vs. mucin-positive intrahepatic CCA2, (ii) IDH mutation status and (iii) clinical outcomes including adjuvant chemotherapy response and disease-free survival. It is anticipated that this will yield new insights into the molecular pathology of cholangiocarcinoma and potentially novel therapeutic strategies. Moreover, molecular signatures predictive for clinical outcomes may form the base of personalized treatment. Chen et al [2] have shown that MALDI MS analysis of lipids distinguishes cancerous epithelium of cholangiocarcinoma from adjacent normal tissue, and between cholangiocarcinomas and colorectal cancers. Here we take this molecular based classification to another level through the direct application of imaging MS on a combination of larger tissue microarrays (figure 1), selected sections of patient tumors and xenografts

\section{References:}

[1] K. Chughtai and Ron M.A. Heeren, Chem. Reviews 110 (2010), p. 3237-3277.

[2] Chen et al., Clin Chim Acta. 412 (2011) p. 1978-1982

[3] The authors acknowledge funding from the regional government of the province of Limburg, the Netherlands through the LINK program as well as the kind technical support from Waters Inc. and Bruker Daltonik GMBH. Dr. Tiffany Porta acknowledges support from the Swiss National Science Foundation within the "SNSF Early Postdoc.Mobility-Fellowships” program (grant P2GEP2_148527).

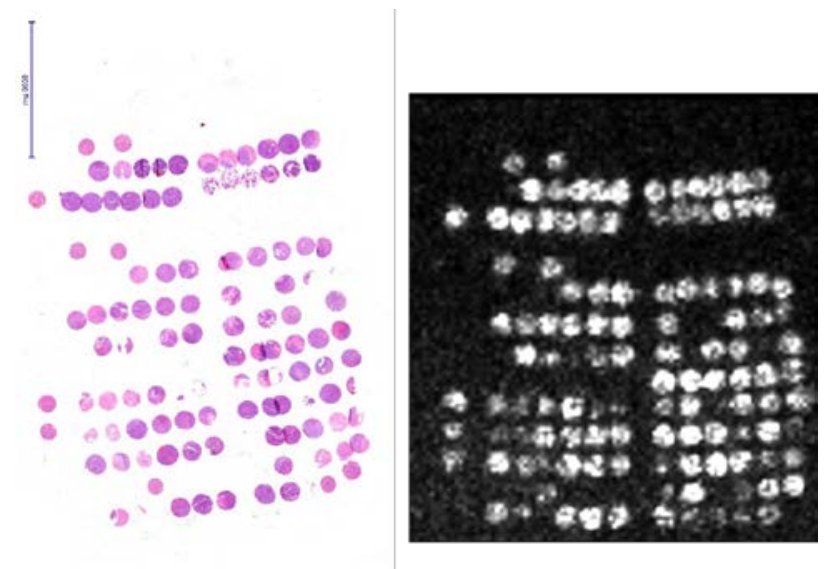

Figure 1. Hematoxylin and eosin stained tissue microarray of patient-derived xenograft models of breast cancer with a corresponding characteristic tryptic peptide ion image at m/z 3187.4. 